\title{
Vigilância do óbito fetal: estudo das principais causas
}

\author{
Death of fetal surveillance: main causes of study
}

\author{
América Maria Eleutério Dell Menezzi* \\ Isabella Drumond Figueiredo** \\ Estefânia Wanderley Barbosa Lima** \\ Júlio César de Almeida** \\ Fúlvia Karine Santos Marques** \\ Camila Ferreira de Oliveira** \\ Nair Amélia Prates Barreto** \\ Lucinéia de Pinho**
}

\begin{abstract}
Resumo
O óbito fetal é uma das principais causas de mortalidade perinatal, e o entendimento das suas causas é essencial para melhorar a assistência pré-natal oferecida às gestantes. Nesse sentido, o estudo caracterizou os casos de óbito fetal intra-útero anteparto registrados em uma maternidade no Norte de Minas entre janeiro de 2010 e dezembro de 2012. Foram avaliadas fichas médicas obtidas junto ao Comitê de Mortalidade Infantil da Maternidade. Foram incluídas na amostragem 26 fichas de gestantes com diagnóstico de óbito fetal ocorrido antes do início do trabalho de parto, com idade gestacional igual ou superior a 20 semanas e ou peso fetal maior que $500 \mathrm{~g}$. Os dados foram coletados por meio de questionário estruturado e avaliados por estatística descritiva. A maioria das gestantes tinha entre 18 e 29 anos e apresentavam gestação a termo. Observou-se que $69 \%$ dos casos de óbito não apresentaram nenhum fator de risco associado, o que é um índice superior aos apresentados na literatura médica. Nos casos com causas identificáveis, atribuiu-se $7,7 \%$ das ocorrências a doenças hipertensivas específicas da gestação, 3,8\% a diabetes mellitus, 3,8\% a infecção do trato urinário, 3,8\% a polidrâmnio e 11,5\% a múltiplas intercorrências. A alta frequência de óbitos sem fatores de risco associados indica que é baixa qualidade da informação sobre o óbito perinatal, especialmente o óbito fetal.
\end{abstract}

Palavras-chave: Óbito fetal. Gestação. Anteparto.

\begin{abstract}
The stillbirth is a major cause of perinatal mortality, and understanding its causes is essential to improve prenatal care provided to pregnant women. In this sense, the study characterized the cases of antepartum intrauterine fetal death recorded in a maternity ward in the North of Minas Gerais between January 2010 and December 2012. Medical records obtained were evaluated by the Child Mortality Committee of Motherhood. They were included in the sample 26 records of pregnant women with fetal death diagnosis occurred before the onset of labor, gestational age less than 20 weeks and birth weight or greater than 500g. Data were collected using a structured questionnaire and evaluated by descriptive statistics. Most of the women were between 18 and 29 years and had a pregnancy to term. It was observed that $69 \%$ of cases of death had no associated risk factor, which is a higher rate to those presented in the medical literature. In cases with identifiable causes, attributed to $7.7 \%$ of cases the specific hypertensive disorders of pregnancy, $3.8 \%$ diabetes mellitus, $3.8 \%$ to urinary tract infection, $3.8 \%$ to 11.5 and polyhydramnios $11,5 \%$ multiple complications. The high frequency of deaths without risk factors indicates that poor quality of information on perinatal death, especially fetal death.
\end{abstract}

Keywords: Stillbirth. Pregnancy. Antepartum.

DOI: $10.15343 / 0104-7809.20164002208212$

*Departamento de Fisiopatologia. Universidade Estadual de Montes Claros - Unimontes. Montes Claros, MG, Brasil. E-mail: amariaeleuterio@gmail.com

** Departamento de Fisiopatologia. Universidade Estadual de Montes Claros - Unimontes. Montes Claros, MG, Brasil

Os autores declaram não haver conflitos de interesse. 


\section{INTRODUÇÃO}

O óbito fetal, que corresponde à morte do feto com mais de 500g ou 22 semanas de gestação ${ }^{1}$, pode trazer à grávida transtornos clínicos, obstétricos e emocionais ${ }^{2,3}$. Apenas em 2015 2,6 milhões de gestações foram interrompidas no mundo por causa de óbito fetal ${ }^{4}$. O óbito fetal ocorre maioritariamente em países de baixa e média renda, sendo um importante indicativo da saúde materno infantil. Enquanto em países desenvolvidos a frequência é de 2 a 7 óbitos fetais a cada mil gestações, no Brasil esse número atingiu 10,97 em 2010 5,6.

As causas do óbito fetal incluem infecções maternas na gestação, doenças maternas, incluindo sífilis, soropositividade com baixa contagem de $\mathrm{CD}^{+}$, malária, diabetes e hipertensão, anomalias congênitas, asfixia e trauma do nascimento, complicações placentárias, umbilicais, amnióticas, uterinas e restrição do crescimento fetal ${ }^{5-8}$.

A infecção neonatal, intimamente relacionada com infecções maternas, também é apontada como uma das principais causas de óbito fetal e neonatal ${ }^{9}$.

Além disso, o óbito fetal pode estar relacionado à pobreza e falta de educação, sobrepeso e idade materna ( $>35$ ou $<20$ anos), paridade $(1, \geq 5)$, tabagismo, falta de cuidados pré-natais, e prevalência de natimorto em gestação ${ }^{7,8}$.

Conhecer a epidemiologia da morte fetal é fundamental para promoção de ações voltadas à saúde materno-infantil, pois a maior visibilidade desse problema e identificação dos fatores determinantes dos óbitos são necessárias para subsidiar a adoção de medidas preventivas que permitam um enfrentamento mais efetivo de um problema que pode ser evitado ${ }^{4,10}$.

Um obstáculo para isso é a frequente limitação da medicina em determinar a causa do óbito fetal. Assim a necessidade de mais estudos sobre esse tema a fim de ter mais segurança nos diagnósticos além de melhorar os processos de conduta e apoio junto à família. Nesse sentido, o presente estudo foi desenvolvido para caracterizar o óbito fetal anteparto, em uma maternidade no Norte de Minas.

\section{MÉTODOS}

O estudo descritivo e transversal consistiu na investigação de fichas médicas de 26 gestantes com óbito fetal anteparto atendidas em um hospital no Norte de Minas Gerais no período de primeiro de janeiro de 2010 a 31 de dezembro de 2012.

Os dados foram coletados de forma retrospectiva, utilizando as declarações de óbito e os prontuários maternos obtidos junto ao Comitê de Mortalidade Infantil do hospital. Foram incluídas no estudo fichas de gestantes com diagnóstico de óbito fetal ocorrido antes do início do trabalho de parto, com idade gestacional igual ou superior a 20 semanas e ou peso fetal maior que $500 \mathrm{~g}$.

Para análise dos documentos utilizou-se um questionário estruturado sobre variáveis clínicoepidemiológicas maternas, incluindo idade materna, idade gestacional, gestação múltipla, polidrâmnio, desnutrição, álcool/drogas, peso ao nascimento, circular de cordão, tipo de parto, motivo da internação, hipertensão arterial crônica/pré-eclâmpsia, diabetes mellitus, diabetes mellitus gestacional, infecção do trato urinário, outras infecções, hemorragias (descolamento prematuro de placenta, placenta prévia) e aspecto do líquido amniótico.

A análise dos dados foi conduzida em planilha eletrônica (Microsoft Office Excel $®$ ) e pelo software SPSS (Statistical Package for Social Sciences) versão 18.0. Os resultados foram descritos por frequência simples.

Antes de ser desenvolvida, pesquisa foi analisada e aprovado pelo Comitê de Ética em Pesquisa da Universidade Estadual de Montes Claros, MG, sob o Parecer n.ํ 2159/2010.

\section{RESULTADOS}

Das fichas-resumo avaliadas, 26 atenderam aos critérios de inclusão estabelecidos. A tabela 1 mostra a frequência de óbitos fetais de acordo com a faixa etária materna, indicando predomínio de ocorrência em gestantes com idade inferior a 30 anos. 
Tabela 1 - Idade materna das gestantes participantes do estudo. Montes Claros, 2012.

A maior parte dos óbitos $(65,4 \%)$ ocorreu principalmente após 37 semanas de gestação, e na maioria dos casos não foi possível detectar nenhum fator de risco associado $(69,2 \%)$. Entre as intercorrências gestacionais detectadas, $11,5 \%$ corresponderam a múltiplas intercorrências, o que dificulta a determinação da etiologia específica do óbito fetal (Tabela 2).

Tabela 2 - Intercorrência gestacionais em casos de morte fetal. Montes Claros, 2012.

\begin{tabular}{lc}
\hline \multicolumn{1}{c}{$\begin{array}{c}\text { Intercorrências } \\
\text { gestacionais }\end{array}$} & $\%$ \\
\hline Sem fatores de risco & 69,4 \\
Múltiplas intercorrências & 11,5 \\
Hipertensão/ & 7,7 \\
Pré-eclâmpsia & \\
Diabetes mellitus \\
(gestacional ou não) \\
Infecção do trato urinário & 3,8 \\
Polidrâmnio & 3,8 \\
\hline
\end{tabular}

Nenhuma gestante apresentou quadro compatível com Data Provável do Parto (DPP). Além da infecção de trato urinário, não foram detectadas outras infecções.

É oportuno destacar que não houve nenhum relato de desnutrição materna e de abuso de substâncias como álcool ou outras drogas ilícitas.

A indução do trabalho de parto correspondeu à metade dos casos de internação, seguido de trabalho de parto a termo, trabalho de parto pré-termo, cesárea eletiva e amniorrexe (Tabela $3)$.
Tabela 3 - Motivo da internação em casos de morte fetal. Montes Claros, 2012.

\begin{tabular}{lc}
\hline \multicolumn{1}{c}{ Motivo da internação } & \% \\
\hline Indução do trabalho de parto & 50,1 \\
Trabalho de parto a termo & 19,2 \\
Trabalho de parto pré-termo & 15,4 \\
Cesárea eletiva & 11,5 \\
Amniorrexe prematura & 3,8 \\
\hline
\end{tabular}

A maioria dos partos $(69,25 \%)$ ocorreu por via vaginal e apenas $26,9 \%$ via cesárea. A circular de cordão foi registrada em 30,8\% dos fetos mortos.

\section{DISCUSSÃO}

O presente estudo foi motivado pelo interesse no conhecimento das causas de óbito fetal em gestantes internadas em um hospital no Norte de Minas Gerais. O tamanho da amostra analisada, com apenas 26 casos, pode ter se configurado como um fator limitador na análise de algumas variáveis selecionadas. Ainda assim, a contribuição do mesmo é significante visto que, talvez pelo sub-registro de óbitos fetais, estudos dessa natureza ainda são escassos no Brasil ${ }^{4}$.

A Portaria $n^{\circ} 72$ do MS, de 11 de janeiro de $2010^{10}$, estabelece a obrigatoriedade da vigilância do óbito infantil e fetal nos serviços que integram o Sistema Único de Saúde (SUS). As notificações devem ter todos os campos preenchidos adequadamente, com informações retiradas da Declaração de Óbito (DO) do feto, a fim de que o Ministério da Saúde alimente sua base de dados e possibilite a investigação dos $\operatorname{casos}^{11}$. Baseado nessa portaria, era de se esperar que houvesse mais informações a respeito das causas de óbito fetal, o que infelizmente não se verifica na prática. Estima-se que a morte fetal seja um fenômeno inexplicável em aproximadamente $60 \%$ dos casos, e muitas vezes não pode ser atribuído a causas maternas, fetais ou obstétricas ${ }^{12}$. No presente estudo, o índice de causas indeterminadas encontrado foi ainda mais alto, atingindo 69,2\%.

Quando identificada a causa do óbito fetal, observa-se que a frequência das várias etiologias 
associadas difere entre países desenvolvidos e em desenvolvimento ${ }^{13}$. Por exemplo, em países em desenvolvimento, pré-eclâmpsia e infecções são causas comuns de óbito fetal, enquanto em países desenvolvidos, a maioria dos casos são relacionados a anomalias congênitas, problemas da placenta e doenças maternas ${ }^{14}$. No presente estudo, a maioria dos óbitos cujas causas foram identificadas foram atribuídos a múltiplas intercorrências, o que é também é de certa forma inespecífico. A principal doença identificada foi a hipertensão/pré-eclampsia, correspondendo a 7,7\% dos casos avaliados. Um estudo com gestantes de Florianópolis, SC, também indicou a doença hipertensiva como razão de $16,4 \%$ dos casos de óbito fetal registrados em um hospital universitário ${ }^{11}$.

Com exceção de um único caso de infecção do trato urinário, não foram verificadas outras infecções maternas e intrauterinas nas fichas avaliadas. Ainda assim, a infecção é tida como causa importante de morte fetal em países em

\section{CONCLUSÃO}

A despeito do atual avanço tecnológico na área médica, o óbito fetal não é uma entidade rara e representa ainda grande proporção da taxa de mortalidade perinatal ${ }^{16}$. Porém, o registro de óbitos fetais tem sido historicamente negligenciado pelos serviços de saúde, que ainda não incorporaram na sua rotina de trabalho a análise de sua ocorrência e tampouco destinaram investimentos específicos para a sua redução ${ }^{17}$. Sua importância como um problema de saúde pública é evidente, mas até mesmo dados epidemiológicos são falhos ${ }^{4}$. Essa é uma séria negligência visto que muitos óbitos fetais poderiam ser evitados, especialmente quando causados por doenças maternas.

Os resultados do presente estudo, que reportam mais de $69 \%$ de óbitos sem fatores de risco associados e mais de 11\% relacionados a múltiplas intercorrências, reforçam a necessidade de investigações mais profundas sobre esses eventos. O profissional de medicina deve ainda ter ciência nos casos onde a morte intrauterina é uma fatalidade sem causa especifica se faz necessário esclarecer aos pais sobre essa verdade e acalmá-los no desenvolvimento ${ }^{11}$.

Não houve no presente estudo nenhum caso de descolamento prematuro de placenta apesar de alguns estudos apontarem esta causa como fator de risco importante. Em relação aos acidentes com o cordão umbilical, é relevante citar que a circular de cordão foi verificada em 30,8\% dos fetos. A prematuridade também é referida como importante fator associado à mortalidade neonatal, podendo ser explicada por situações maternas graves que podem comprometer o desenvolvimento do feto e levar à necessidade de interrupção prematura da gestação .

Outros estudos associam o óbito fetal ao uso de drogas, álcool e tabaco, mas esses hábitos não constavam em nenhuma das fichas das gestantes avaliadas. Quanto à idade, as faixas extremas são consideradas como fatores de risco para o óbito fetal ${ }^{15}$. Na população estudada, $35 \%$ das gestantes encontravam-se nas idades de risco.

momento do diagnóstico e em relação às futuras gestações.

Para o Brasil, e em particular, para o hospital onde foi conduzido o estudo, colocase o desafio de melhor qualificar a informação sobre o óbito perinatal, especialmente sobre o óbito fetal, incorporando essa prática à rotina dos serviços. Somente com o incentivo para o registro adequado da informação e o contínuo retorno e avaliação das informações produzidas pelos serviços de saúde poderá haver a qualificação do mesmo, de modo a melhorar a compreensão da ocorrência dos óbitos fetais e, consequentemente, melhorar o controle e prevenção dos eventos considerados evitáveis.

Quanto às causas de óbito fetal identificadas, embora tenham representado uma parcela menor dos casos, ainda assim servem de base para delinear planos de prevenção junto às gestantes, principalmente na assistência prénatal.

Os programas desenvolvidos devem enfatizar a necessidade de acompanhamento médico e tratamento continuado de condições patológicas que podem ocasionar o óbito fetal. 


\section{REFERÊNCIAS}

1. Organização Mundial da Saúde. Classificação estatística internacional de doenças e problemas relacionados à saúde, $10 a$ revisão. 8a Ed. São Paulo: Edusp; 2008.

2. Duarte CAM, Turato ER. Sentimentos presentes nas mulheres diante da perda fetal: uma revisão. Psicol Estud. 2009;14(3):485-490.

3. Scott J. Stillbirths: breaking the silence of a hidden grief. Lancet. 2011;377(9775):1386-1388.

4. Barbeiro FMS, Fonseca SC, Tauffer MG, Ferreira MSS, Silva FP, Ventura PM et al. Óbitos fetais no Brasil: revisão sistemática. Rev Saúde Pública. 2015;49(22):1-13.

5. Cousens S, Blencowe H, Stanton C, Chou D, Ahmed S, Steinhardt L, et al. National, regional, and worldwide estimates of stillbirth rates in 2009 with trends since 1995: a systematic analysis. The Lancet. 2011;16(377):1319-1330.

6. Lawn JE, Blencowe H, Pattinson R, Cousens S, Kumar R, Ibiebele I, et al. Stillbirths: Where? When? Why? How to make the data count? The Lancet. 2011;16(377):1448-1463.

7. Aminu M, Unkels R, Mdegela M, Utz B, Adaji S, van den Broek N. Causes of and factors associated with stillbirth in low- and middleincome countries: a systematic literaturereview. BJOG. 2014;121(suppl. 4):141-153.

8. Quibel T, Bultez T, Nizard J, Subtil D, Huchon C, Rozenberg P. In utero morte fetal. J Gynecol Obstet Biol Reprod. 2014;43(10):883907.

9. Oliveira LC, Costa AAR. Óbitos fetais e neonatais entre casos de near miss materno. Rev Assoc Med Bras. 2013;59(5):487-494.

10. Brasil. Ministério da Saúde. Portaria GM № 72, de 11 de Janeiro de 2010. Estabelece que a vigilância do óbito infantil e fetal é obrigatória nos serviços de saúde (públicos e privados) que integram o Sistema Único de Saúde (SUS). Disponível em: http://bvsms. saude.gov.br/bvs/saudelegis/gm/2010/prt0072_11_01_2010.html. Acesso em: 03 Jun 2016.

11. Vieira MSM, Siebert EC, Ceglio WQGW, Almeira MH, Batista TS. Dificuldades para a identificação da causa do óbito fetal: como resolver? Rev Bras Ginecol Obstet. 2012;34(9): 403-409.

12. Bukowski R, Carpenter M, Conway D, Coustan D, Dudley DJ, Goldenberg RL, et al. Collaborative research network writing group: causes of death among stillbirths. JAMA. 2011;306(22):2459-2468.

13. Silver RM, Varner MW, Reddy U, Goldenberg R, Pinar H, Conway D, et al. Work-up of stillbirth: a review of the evidence. Am J Obstet Gynecol. 2007;196(5):433-444.

14. Mcclurem EM, Nalubamba-Phiri M, Goldenberg RL. Stillbirth in developing countries. Int J Gynaecol Obstet. 2006;94(2):82-90.

15. Patel EM, Goodnight WH, James AH, Grotegut CA. Temporal trends in maternal medical conditions and stillbirth. Am J Obstet Gynecol. 2015; 212(5):673.e1-11.

16. Jacinto E, Aquino EML, MOTA ELA. Mortalidade perinatal no município de Salvador, Bahia: evolução de 2000 a 2009 . Rev Saúde Pública. 2013;47(5):846-853. 